\title{
Serum cytokine biomarker panels for discriminating pancreatic cancer from benign pancreatic disease
}

\author{
Victoria E Shaw ${ }^{\dagger}$, Brian Lane ${ }^{\dagger}$, Claire Jenkinson, Trevor Cox, William Greenhalf, Christopher M Halloran,
} Joseph Tang, Robert Sutton, John P Neoptolemos and Eithne Costello*

\begin{abstract}
Background: We investigated whether combinations of serum cytokines, used with logistic disease predictor models, could facilitate the detection of pancreatic ductal adenocarcinoma (PDAC).

Methods: The serum levels of 27 cytokines were measured in 241 subjects, 127 with PDAC, 49 with chronic pancreatitis, 20 with benign biliary obstruction and 45 healthy controls. Samples were split randomly into independent training and test sets. Cytokine biomarker panels were selected by identifying the top performing cytokines in best fit logistic regression models during multiple rounds of resampling from the training dataset. Disease prediction by logistic models, built using the resulting cytokine panels, was evaluated with training and test sets and further examined using resampled performance evaluation.

Results: For the discrimination of PDAC patients from patients with benign disease, a panel of IP-10, IL-6, PDGF plus CA19-9 offered improved diagnostic performance over CA19-9 alone in the training (AUC 0.838 vs. 0.678) and independent test set (AUC 0.884 vs. 0.798). For the discrimination of PDAC from CP, a panel of IL-8, CA19-9, IL-6 and IP-10 offered improved diagnostic performance over CA19-9 alone with the training (AUC 0.880 vs. 0.758 ) and test set (AUC 0.912 vs. 0.848). Finally, for the discrimination of PDAC in the presence of jaundice from benign controls with jaundice, a panel of IP-10, IL-8, IL-1b and PDGF demonstrated improvement over CA19-9 in the training (AUC 0.810 vs. 0.614 ) and test set (AUC 0.857 vs. 0.659).
\end{abstract}

Conclusions: These findings support the potential role for cytokine panels in the discrimination of PDAC from patients with benign pancreatic diseases and warrant additional study.

Keywords: Pancreatic cancer, Biomarker, Cytokine, CA19-9, IP-10, IL-8 and PDGF

\section{Introduction}

Novel biomarkers for use in disease detection and/or treatment are urgently needed to improve outcomes for patients with pancreatic cancer (PDAC) [1,2]. Supplementing current diagnostic modalities with biomarker detection in blood [3] could potentially enhance PDAC diagnosis. At present, the only serum biomarker in routine clinical use for PDAC is CA19-9 [4-6]. The ability of novel biomarkers to accurately detect PDAC depends on their capacity to

\footnotetext{
* Correspondence: ecostell@liverpool.ac.uk

${ }^{\dagger}$ Equal contributors

NIHR Liverpool Pancreas Biomedical Research Unit, Royal Liverpool and Broadgreen University Hospital NHS Trust, Department of Molecular and Clinical Cancer Medicine, University of Liverpool, Liverpool L69 3GA, UK
}

discriminate PDAC from benign diseases of the pancreas, such as chronic pancreatitis. In addition, a majority of PDAC patients present with tumours involving the pancreatic head, which leads to obstructive jaundice [7]. The differentiation of PDAC in jaundiced patients from benign obstructive jaundice due to choledocholithiasis or chronic pancreatitis is a major clinical challenge.

CA19-9 is a sialyated Lewis blood group cell surface carbohydrate antigen, expressed in normal pancreatic ductal cells in around 95\% of the population which express the Lewis antigen glycosyltransferase enzyme. CA19-9 is shed into the general circulation and is commonly used in clinical practice to monitor patients with PDAC [4,5,8-10]. CA19-9 is also secreted in a mucin bound form by the 
biliary and gallbladder mucosa and is exclusively excreted in bile [11]. Serum levels of CA19-9 are elevated in patients with chronic pancreatitis and benign biliary obstruction to a similar extent as in patients with smaller pancreatic cancers $[4,8]$. Consequently the overall accuracy of CA19-9 for the diagnosis of PDAC is reduced but there is also the opportunity to enhance the specificity of CA19-9 in combination with other tumour-associated biomarkers [12-17]. Mediators of the tumour microenvironment and the host response [12-15,18] and notably cytokines involved in the immune system, inflammation, tumour development and metastasis $[19,20]$ are emerging as key candidate biomarkers. While single cytokines lack sensitivity and specificity for accurate cancer detection [21], specific combinations may prove valuable as markers.

Cytokine biomarker panels for the discrimination of specific patient groups were selected by identifying the best logistic regression models during multiple rounds of resampling [22] from a training dataset. The resulting optimum panels were evaluated using logistic regression models in both training and independent test sets before further subjecting panels to resampling performance evaluation. We discovered a unique panel of cytokines that improved the performance of CA19-9 for the discrimination of PDAC patients from patients with benign pancreatic disease. Moreover, in the presence of jaundice, whilst CA19-9 offered relatively poor discrimination of PDAC patients from benign disease patients, a panel made up solely of cytokines afforded significantly better discrimination.

\section{Results}

Cytokine levels in patients diagnosed with PDAC, chronic pancreatitis and benign biliary obstruction and healthy subjects

Filtering of the entire dataset showed that serum levels of nine cytokines, comprising PDGF, IL-1b, IL-1ra, IL-6, IL-8, Eotaxin, IP-10, MCP-1 and MIP-1b, were significantly different between PDAC in comparisons with one or more of the control variants (Table 1). Serum levels of five cytokines, IL-1ra, IL-6, IL-8, IP-10 and MIP-1b, as well as serum CA19-9 levels were significantly increased in PDAC compared to HCs. Of these, CA19-9, IL-8 and IP-10, were also significantly elevated in PDAC compared to patients with $\mathrm{CP}$, whilst a comparison of PDAC versus $\mathrm{BBO}$ revealed significant increases in serum levels of Eotaxin, IL-1b, MIP-1b and PDGF (Table 1). Serum levels of CA19-9, IL-8, IP-10, MIP-1b and PDGF, were significantly elevated in patients with PDAC compared to patients with benign disease (Table 1). Comparison of serum cytokine levels in subjects with obstructive jaundice showed that IL-8, IP-10, MIP-1b, PDGF and CA19-9 were all significantly elevated in PDAC compared to controls (Table 2). The circulating median levels of cytokines and CA19-9 (i.e. un-normalised) are shown in Additional file 1: Table S1. Spearman's Rank analysis of the cytokines incorporated into panels and CA19-9 for each group showed a maximum Rho of 0.361 , indicating no correlation between age and analyte level.

\section{Classification model to distinguish patients with PDAC from healthy subjects}

In the training dataset (84 PDAC, $29 \mathrm{HCs}$ ), serum CA19-9 had a very high performance in distinguishing patients with PDAC from healthy subjects $(\mathrm{AUC}=0.925$, $\mathrm{CI}=0.876-0.974$ ). Serum levels of IL-8 and IL-1b were also both found with high frequency in top ranked models of the training set in successive resamples (100\% and 95\% respectively) as well as CA19-9 (99\%) (Figure 1A). The combined panel comprising IL-8, IL-1b and CA19-9 showed a statistically significant improvement in accuracy over CA19-9 alone $(\mathrm{AUC}=0.984, \mathrm{CI}=$ $0.968-1.00$ vs. $\mathrm{AUC}=0.925, \mathrm{CI}=0.876-0.974 ; \mathrm{p}=0.004$; Figure 1B), although diagnostic improvement of the panel over CA19-9 was not statistically significant in the test set $(\mathrm{PDAC}=43, \mathrm{HC}=16)(\mathrm{AUC}=0.997, \mathrm{CI}=0.990$ 1.00 vs. $\mathrm{AUC}=0.975, \mathrm{CI}=0.932-1.00)$ (Figure $1 \mathrm{C}$ ). Resampling the combined dataset $(\mathrm{PDAC}=127, \mathrm{HC}=45)$ 100 times however, showed a statistically significant improved accuracy for the panel compared to CA19-9 alone $($ median $=94.6 \%, \mathrm{IQR}=93.8-96.4 \%$ vs. median $=$ 89.3\%, IQR $=88.4-91.1 \%$ respectively; Friedman test $\mathrm{p}<$ 0.001). The SN (median $=94.1 \%, \mathrm{IQR}=91.8-96.4 \%$, ver sus $85.9 \%, \mathrm{IQR}=84.7-89.4 \%$ respectively) and SP (median $=100 \%$, IQR $=96.3-100 \%$ versus median $=96.3 \%$, $\mathrm{IQR}=96.3-97.2 \%)$ of the panel on resampling were also significantly higher than resampled CA19-9 alone (both Friedman test $\mathrm{p}<0.001$; Figure 1D).

\section{Classification model to distinguish patients with PDAC from patients with benign disease}

Model building using the training set $(\mathrm{PDAC}=84$, benign disease $=45)$ to distinguish PDAC patients from patients with benign pancreatic disease showed that the most frequent cytokines in top ranked resampled models were IL-8 (98\%), IP-10 (76\%), IL-6 (56\%) and PDGF (36\%) plus CA19-9 (22\%) (Figure 2A). As a panel, these cytokines demonstrated improved diagnostic performance over CA19-9 alone $(\mathrm{AUC}=0.838 ; \mathrm{CI}=0.768-0.909$ vs. $\mathrm{AUC}=0.678 ; \mathrm{CI}=.579-0.776 ; \mathrm{p}<0.001$ ) (Figure $2 \mathrm{~B}$ ) with significantly improved $\mathrm{SN}$ (median $=92.9 \%, \mathrm{CI}=$ $85-97 \%$ vs. median $=53.6 \%, C I=42-65 \%$, respectively, $\mathrm{p}<0.001$ ), but significantly reduced SP (median $=57.8 \%$, $\mathrm{CI}=42-72 \%$ vs. median $=84.4 \%, \mathrm{CI}=71-94 \%$ respectively, $\mathrm{p}=0.008)$. Using the independent test data $(\mathrm{PDAC}=$ 43 , benign disease $=24)$, the panel showed improved performance over CA19-9 alone $(\mathrm{AUC}=0.884, \mathrm{CI}=$ 
Table 1 Normalised cytokine data in the combined training and test datasets

\begin{tabular}{|c|c|c|c|c|c|c|c|c|c|}
\hline \multirow[b]{2}{*}{ Analyte } & \multicolumn{5}{|c|}{ Median (95\% Cl) } & \multicolumn{4}{|c|}{$P$ value } \\
\hline & PDAC $(n=127)$ & $H C(n=45)$ & $C P(n=49)$ & $\mathrm{BBO}(\mathrm{n}=20)$ & $C P+B B O(n=69)$ & PDAC vs. HC & PDAC vs. CP & PDAC vs. BBO & PDAC vs. $C P+B B O$ \\
\hline \multirow[t]{2}{*}{ CA19-9 } & 0.01 & -5.01 & -2.7 & -0.82 & -2.25 & $<0.001$ & $<0.001$ & NS & $<0.001$ \\
\hline & $(-1.0$ to 0.2$)$ & $(-5.4$ to -4.6$)$ & $(-3.5$ to -2.2$)$ & (-2.6 to 0.5$)$ & $(-3.2$ to -1.6$)$ & & & & \\
\hline \multirow[t]{2}{*}{ Eotaxin } & 0.48 & 0.23 & 0.53 & -0.08 & 0.45 & NS & NS & $<0.05$ & NS \\
\hline & (0.3 to 0.6$)$ & $(-0.2$ to 0.5$)$ & (0.2 to 0.8$)$ & $(-0.5$ to 0.7$)$ & $(-0.03$ to 0.7$)$ & & & & \\
\hline \multirow[t]{2}{*}{ IL-1b } & 0.23 & 0.16 & 0.27 & 0.14 & 0.17 & NS & NS & $<0.05$ & NS \\
\hline & (0.1 to 0.4 ) & (0.08 to 0.3$)$ & (0.04 to 0.6$)$ & $(-0.5$ to 0.5$)$ & (0.05 to 0.4$)$ & & & & \\
\hline \multirow[t]{2}{*}{ IL-1ra } & 0.72 & 0.18 & 0.78 & 0.06 & 0.59 & $<0.05$ & NS & NS & NS \\
\hline & (0.4 to 1.0$)$ & $(-0.3$ to 0.4$)$ & (0.4 to 1.3 ) & $(-1.5$ to 1.5$)$ & (0.2 to 1.0$)$ & & & & \\
\hline \multirow[t]{2}{*}{ IL-6 } & 1.25 & 0.41 & 1.65 & 1.78 & 1.71 & $<0.001$ & NS & NS & NS \\
\hline & (1.0 to 1.5$)$ & (0.1 to 0.7 ) & (1.2 to 2.1$)$ & (1.0 to 2.5$)$ & (1.2 to 2.0$)$ & & & & \\
\hline \multirow[t]{2}{*}{ IL-8 } & 0.95 & 0.02 & 0.54 & 0.38 & 0.51 & $<0.001$ & $<0.001$ & NS & $<0.001$ \\
\hline & (0.8 to 1.2 ) & $(-0.3$ to 0.06$)$ & (0.3 to 0.8 ) & (-0.4 to 1.1$)$ & (0.3 to 0.8 ) & & & & \\
\hline \multirow[t]{2}{*}{ IP-10 } & -1.85 & -2.99 & -2.6 & -2.52 & -2.59 & $<0.001$ & $<0.001$ & NS & $<0.001$ \\
\hline & $(-2.0$ to -1.7$)$ & $(-3.3$ to -2.7$)$ & $(-2.9$ to -2.3$)$ & $(-2.9$ to -1.5$)$ & $(-2.8$ to -2.3$)$ & & & & \\
\hline \multirow[t]{2}{*}{ MCP-1 } & 0.31 & 0.44 & 0.92 & 0.64 & 0.81 & NS & NS & NS & NS \\
\hline & (0.2 to 0.6$)$ & $(-0.02$ to 0.6$)$ & (0.3 to 1.2$)$ & $(-0.2$ to 1.6$)$ & (0.3 to 1.2$)$ & & & & \\
\hline \multirow[t]{2}{*}{ MIP-1b } & 0.31 & -0.45 & 0.02 & -0.28 & -0.06 & $<0.001$ & NS & $<0.05$ & $<0.01$ \\
\hline & (0.2 to 0.4$)$ & $(-0.6$ to -0.2$)$ & $(-0.3$ to 0.4$)$ & $(-0.5$ to 0.2$)$ & $(-0.3$ to 0.2$)$ & & & & \\
\hline \multirow[t]{2}{*}{ PDGF } & 0.44 & 0.31 & 0.21 & -0.62 & 0.09 & NS & NS & $<0.01$ & $<0.01$ \\
\hline & (0.2 to 0.5 ) & (0.1 to 0.4 ) & $(-0.02$ to 0.5$)$ & $(-1.5$ to 0.2$)$ & $(-0.18$ to 0.4$)$ & & & & \\
\hline
\end{tabular}

Median normalised $\log _{2}$ transformed data are presented for each analyte; the $95 \% \mathrm{Cl}$ are given in brackets. PDAC = Cancer, $\mathrm{CP}=$ Chronic Pancreatitis, $\mathrm{BBO}=\mathrm{Benign} \mathrm{Biliary}$ Obstruction. Wilcoxon signed rank tests were used to generate p-values for group comparisons. NS - non-significant. 
Table 2 Cytokine data from patients with biliary obstruction (cancer and benign) in the combined training and test datasets

\begin{tabular}{|c|c|c|c|c|c|}
\hline Analyte & $\begin{array}{l}\text { Median }(95 \% \mathrm{Cl}) \text { PDAC } \\
\text { with biliary obstruction } \\
\qquad(\mathrm{n}=\mathbf{8 3})\end{array}$ & $\begin{array}{l}\text { Median }(95 \% \mathrm{Cl}) \text { BBO plus } \\
\text { CP with biliary obstruction } \\
\qquad(\mathrm{n}=27)\end{array}$ & $\begin{array}{c}\text { P Value } \\
\text { PDAC with biliary obstruction vs. } \\
\text { Obstructed BBO plus CP with biliary } \\
\text { obstruction }\end{array}$ & $\begin{array}{c}\text { Median }(95 \% \mathrm{Cl}) \text { PDAC } \\
\text { without biliary obstruction } \\
(n=44)\end{array}$ & $\begin{array}{l}\text { P value } \\
\text { PDAC with biliary obstruction vs. PDAC } \\
\text { without biliary obstruction PDAC }\end{array}$ \\
\hline \multirow[t]{2}{*}{ CA19-9 } & 0.16 & -1.10 & $<0.05$ & -1.06 & $<0.05$ \\
\hline & $(-0.6$ to 0.4$)$ & $(-2.3$ to -0.5$)$ & & $(-1.8$ to 0.1$)$ & \\
\hline \multirow[t]{2}{*}{ Eotaxin } & 0.48 & 0.40 & NS & 0.51 & NS \\
\hline & (0.2 to 0.6$)$ & $(-0.3$ to 0.7$)$ & & (0.2 to 0.8$)$ & \\
\hline \multirow[t]{2}{*}{ IL-1b } & 0.23 & 0.25 & NS & 0.24 & NS \\
\hline & (0.1 to 0.4$)$ & (0.03 to 0.4 ) & & (0.002 to 0.4$)$ & \\
\hline \multirow[t]{2}{*}{ IL-1ra } & 0.72 & 0.33 & NS & 0.79 & NS \\
\hline & (0.4 to 1.0 ) & ( -0.8 to 1.0$)$ & & (0.2 to 1.3 ) & \\
\hline \multirow[t]{2}{*}{ IL-6 } & 1.38 & 1.97 & NS & 0.92 & NS \\
\hline & (1.2 to 1.7$)$ & (1.0 to 2.4 ) & & (0.7 to 1.3 ) & \\
\hline \multirow[t]{2}{*}{ IL-8 } & 0.96 & 0.39 & $<0.05$ & 0.89 & NS \\
\hline & (0.8 to 1.4 ) & (0.2 to 1.1$)$ & & (0.5 to 1.2$)$ & \\
\hline \multirow[t]{2}{*}{ IP-10 } & -1.79 & -2.59 & $<0.05$ & -2.06 & NS \\
\hline & $(-2.0$ to -1.6$)$ & $(-2.8$ to -2.2$)$ & & $(-2.5$ to -1.7$)$ & \\
\hline \multirow[t]{2}{*}{ MCP-1 } & 0.26 & 0.56 & NS & 0.40 & NS \\
\hline & (0.05 to 0.6 ) & (0.001 to 1.4$)$ & & (0.08 to 0.8 ) & \\
\hline \multirow[t]{2}{*}{ MIP-1b } & 0.34 & -0.06 & $<0.05$ & 0.29 & NS \\
\hline & (0.2 to 0.5$)$ & ( -0.5 to 0.3$)$ & & $(-0.09$ to 0.35$)$ & \\
\hline \multirow[t]{2}{*}{ PDGF } & 0.42 & -0.27 & $<0.01$ & 0.51 & NS \\
\hline & (0.2 to 0.5 ) & ( -0.9 to 0.4$)$ & & (0.1 to 0.7 ) & \\
\hline
\end{tabular}

Median normalised $\log _{2}$ transformed data are presented for each analyte; the $95 \% \mathrm{Cl}$ are given in brackets. PDAC $=\mathrm{Cancer}, \mathrm{CP}=$ Chronic Pancreatitis, $\mathrm{BBO}=\mathrm{Benign} \mathrm{Biliary}$ Obstruction. Wilcoxon signed rank tests were used to generate p-values for group comparisons. NS - non-significant. 

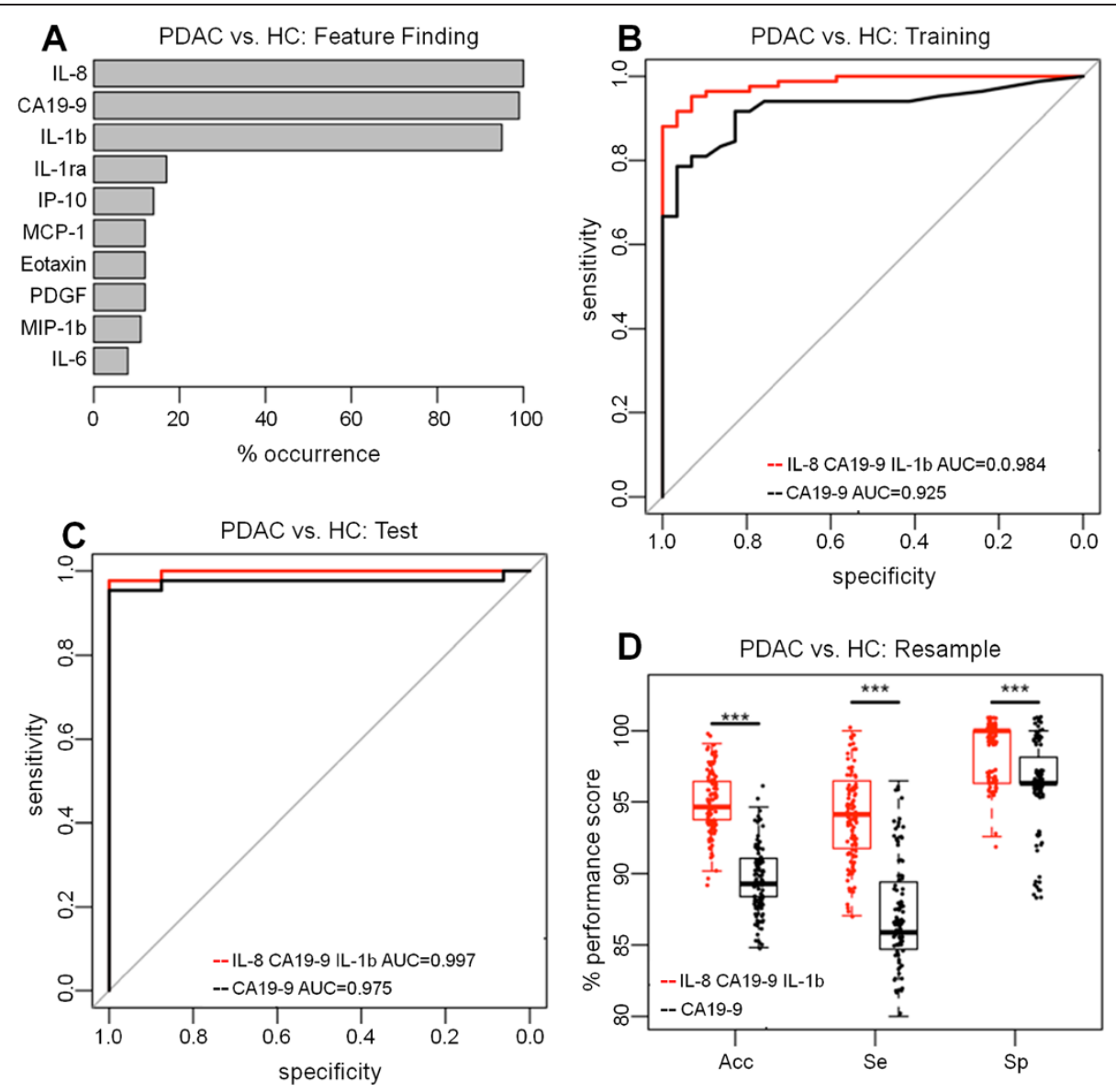

Figure 1 Classification of patients with PDAC vs. healthy control individuals. A. Feature finding showing the percentage occurrence of cytokines in models following resampling of training data for PDAC vs. Healthy Control. B. Training set ROC Curves for PDAC vs. Healthy Control for a panel of IL-8, CA19-9 and IL-1b versus CA19-9 alone. C. Test set ROC curves for PDAC vs. Healthy Control for the panel of IL-8, CA19-9 and $\mathrm{IL}-1 \mathrm{~b}$ versus CA19-9. D. The resampling performance of the panel of IL-8, CA19-9 and IL-1 b versus CA19-9 for the classification of PDAC vs. Healthy Control. The accuracies, sensitivities and specificities of the panel compared to CA19-9 alone over 100 patient-balanced resamples is shown. The quantiles of the performance indicators are summarised as boxplots with individual points superimposed and the significance of Friedman test comparisons is indicated $(* * * 0<0.001)$.

0.802-0.966 vs. $\mathrm{AUC}=0.798, \mathrm{CI} 0.676-0.919$ respectively; $\mathrm{p}=0.058$ ) (Figure 2C) with a median $\mathrm{SN}$ of $81.4 \%(\mathrm{CI}=$ $67-92 \%)$ and SP of $91.7 \%(\mathrm{CI}=73-99 \%)$ for the panel compared to $88.4 \%(\mathrm{CI}=75-96 \%)$ and $66.7 \%(\mathrm{CI}=45$ $84 \%)$ respectively for CA19-9 alone. The inconsistency in the outcomes with training and test set data here illustrate the problems associated with diagnostic performance assessment using single data splits.

Resampling of the combined dataset (PDAC $=127$, benign disease $n=69$ ) (Figure 2D) generated 100 independent optimum values for SN, SP and accuracy and showed that the accuracy of the cytokine panel (median $=77.8 \%$, IQR $=75.6-79.4 \%)$ was significantly improved compared to CA19-9 alone (median $=67.9 \%, \quad \mathrm{IQR}=64.1-72.5 \%$; Friedman test $\mathrm{p}<0.001)$. The SP of the cytokine panel (median $=84.8 \%$, IQR $=80.4-89.1 \%$ ) was significantly better than that of CA19-9 alone (median $=78.2 \%$, $\mathrm{IQR}=58.7-84.8 \%$; Friedman test $\mathrm{p}<0.001)$, while the improvement in SN was not statistically significant ( median $=74.7 \%$, IQR $70.6-77.9 \%$ vs. median $=58.8 \%$, $\mathrm{IQR}=52.9-80.0 \%$ respectively; Friedman test $\mathrm{p}=0.071$ ) (Figure 2D).

\section{Classification model to distinguish patients with PDAC from patients with chronic pancreatitis}

Optimum cytokine combinations for the discrimination of PDAC from $\mathrm{CP}$ with the training set $(\mathrm{PDAC}=84$ and $\mathrm{CP}=32$ ) showed that the most frequent cytokines in resampled models were IL-8 (97\%), IL-6 (63\%) and IP-10 (54\%) as well as CA19-9 (77\%) (Figure 3A). A panel comprising IL-8, IL-6 and IP-10 with CA19-9, showed a significantly improved diagnostic performance with the training data over CA19-9 alone (AUC $=0.880, \mathrm{CI}=0.818-0.943$ vs. $\mathrm{AUC}=0.758, \mathrm{CI}=0.668-0.849 ; \mathrm{p}=0.005$ ) (Figure $3 \mathrm{~B}$ ). The SN of the panel (median $=75.0 \%, C I=64-84 \%$ ) was significantly better than CA19-9 alone $($ median $=53.6 \%$, 

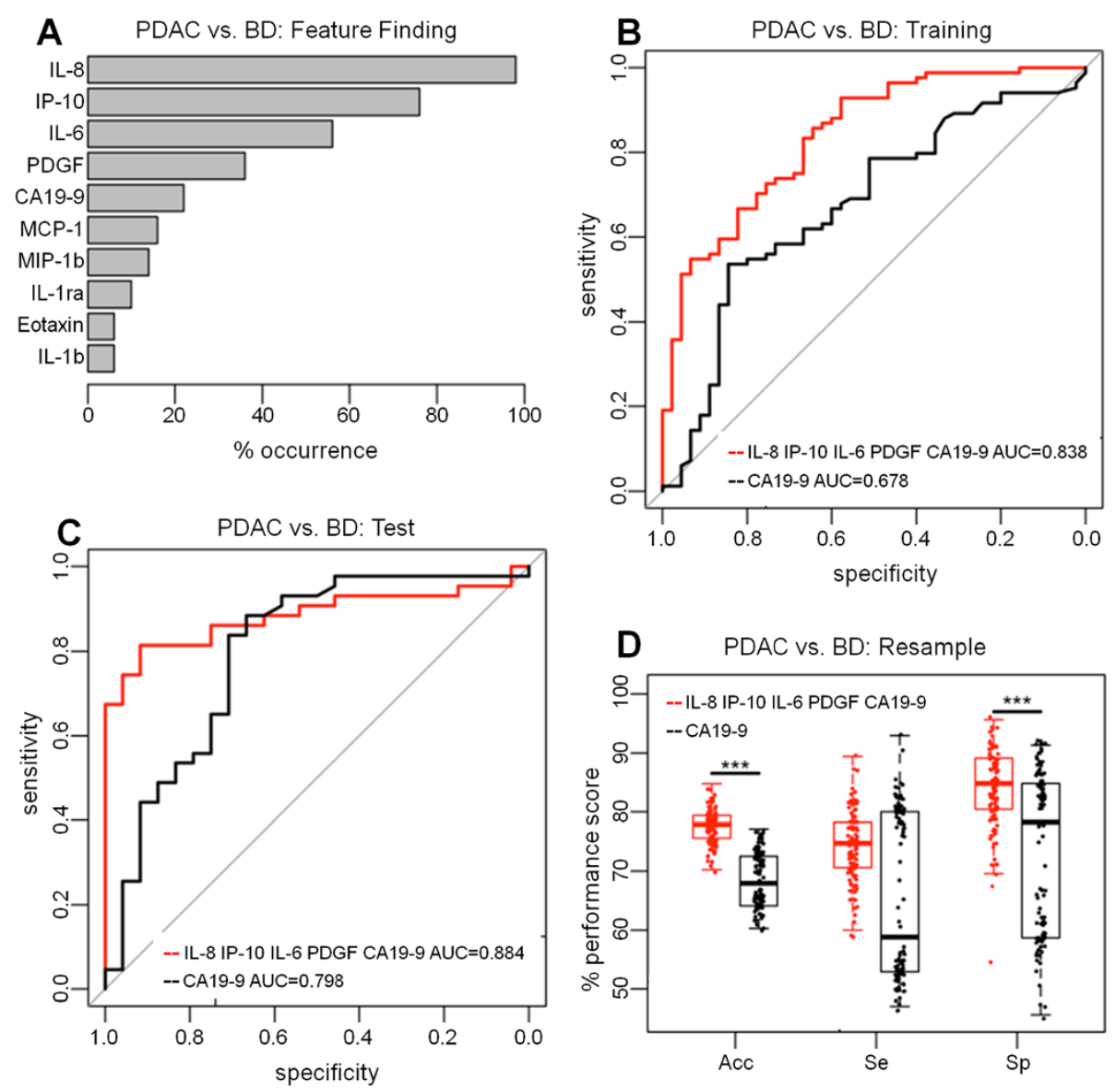

Figure 2 Classification of patients with PDAC vs. patients with benign disease. (A) Feature finding results showing the percentage occurrence of cytokines in models following resampling of training data for PDAC vs. benign disease. (B) Training set ROC Curves for PDAC vs. benign disease for a panel of IL-8, IP-10, IL-6, PDGF and CA19-9 versus CA19-9 alone. (C) Test set ROC curves for PDAC vs. benign disease for the panel of IL-8, IP-10, IL-6, PDGF versus CA19-9. (D) The resampling performance of the panel of IL-8, IP-10, IL-6, PDGF and CA19-9 versus CA19-9 for the classification of PDAC vs. benign disease. The accuracies, sensitivities and specificities of the panel compared to CA19-9 alone over 100 patient-balanced resamples is shown. The quantiles of the performance indicators are summarised as boxplots with individual points superimposed and the significance of Friedman test comparisons is indicated $\left({ }^{* *} \mathrm{p}<<0.001\right)$.

$\mathrm{CI}=42-65 \% ; \mathrm{p}<0.001)$ whilst maintaining a high SP ( median $=90.6 \%, \mathrm{CI}=75-98 \%$ vs. median $=96.9 \%, \mathrm{CI}=$ $84-100 \%$, respectively). In the independent test set ( $\mathrm{PDAC}=43, \mathrm{CP}=17$ ), the AUC achieved with the panel was greater than that of CA19-9 (0.912; CI 0.838-0.987 vs. AUC 0.848; CI 0.728-0.968, respectively) (Figure 3C).

Resampling the combined dataset (PDAC $=127$ and $\mathrm{CP}=49$ ) revealed a significant improvement in accuracy with the cytokine panel (median $=79.5 \%$, IQR $=77.8$ $82.1 \%$ ) compared to CA19-9 (median $=73.5 \%$, IQR $=$ 64.7-76.1\%; Friedman test $\mathrm{p}<0.001$ ) (Figure 3D). The SP was also significantly better (median $=90.9 \%, \quad \mathrm{IQR}=$ $87.9-93.9 \%$ vs. median $=78.8 \%$, IQR $=69.7-93.9 \%$ respectively; Friedman test $\mathrm{p}<0.001)$ with no significant difference in SN estimates (median $=76.2 \%$, IQR $=72.6$ $78.6 \%$ vs. median $=73.8 \%$, IQR $=52.3-78.6 \%$, respectively; Friedman test $\mathrm{p}=0.053$ ).
Classification model in patients with obstructive jaundice to distinguish patients with PDAC from patients with benign disease

The training dataset of patients with serum bilirubin levels $\geq 20 \mu \mathrm{mol} / \mathrm{L} \quad(\mathrm{PDAC}=55$, benign disease = 18) was resampled and the top ranked regression models most frequently featured were IP-10 (65.4\%), IL-8 (50.0\%), IL-1b (41.0\%) and PDGF (41.0\%) whilst CA19-9 was the feature of lowest importance (6.1\%) (Figure 4A). The cytokine panel (IP-10,IL-8,IL-1b and PDGF) was significantly more accurate than CA19-9 (AUC $=0.810, \mathrm{CI}=0.693-0.927$ vs. $\mathrm{AUC}=0.614, \mathrm{CI}=0.445-0.782 ; \mathrm{p}=0.023$ ) (Figure 4B) with significantly improved SN (median $=87.3 \%, \mathrm{CI}=76-95 \%$ vs. median $=63.6 \%, C I=50-76 \%$ respectively; $\mathrm{p}<0.001)$ and unchanged SP $($ median $=66.7 \%, \mathrm{CI}=41-87 \%$ vs. median $=$ $66.7 \%, \mathrm{CI}=41-87 \%$, respectively). The test set of samples with serum bilirubin levels $\geq 20 \mu \mathrm{mol} / \mathrm{L} \quad(\mathrm{PDAC}=28$, 

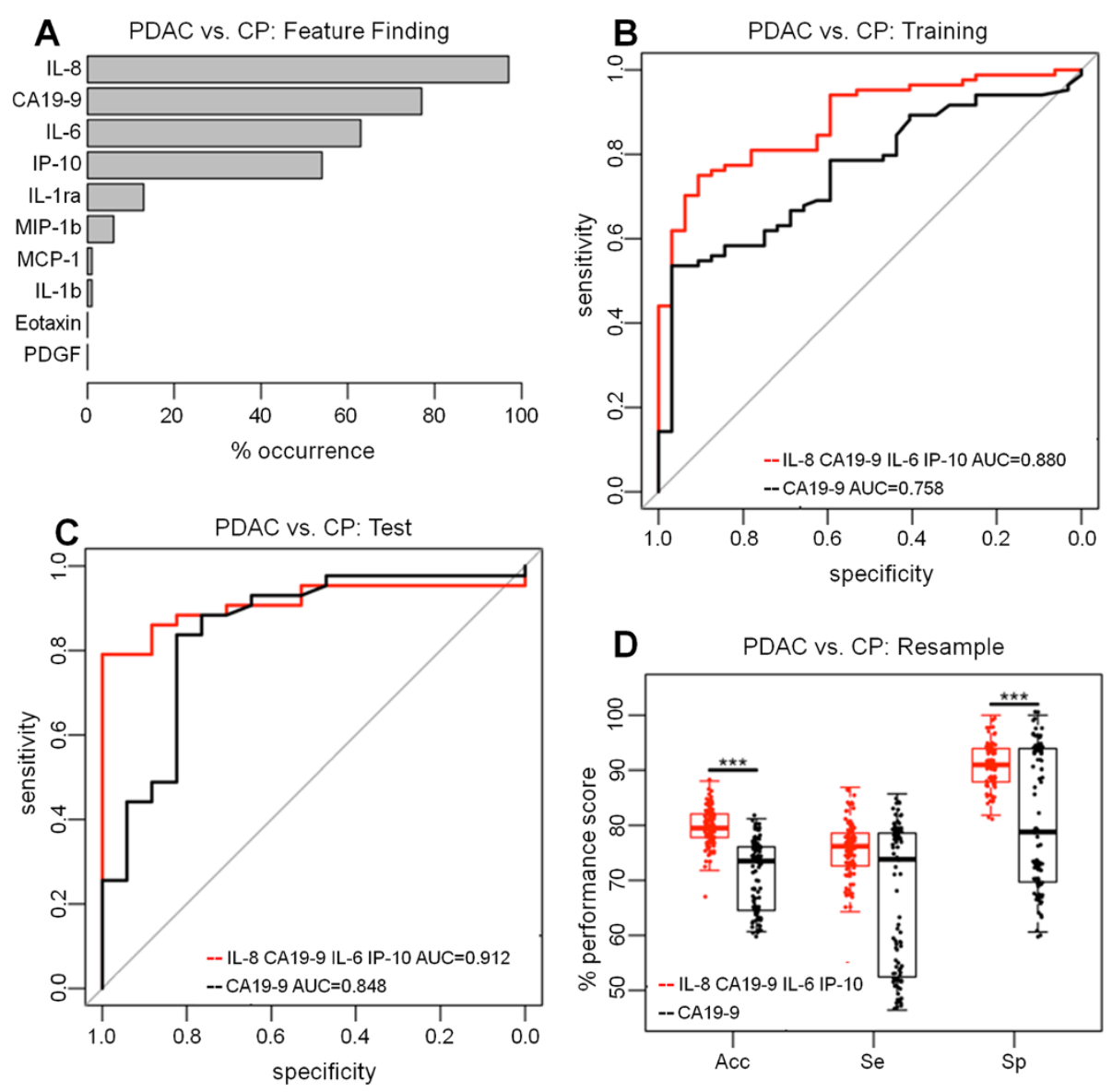

Figure 3 Classification of patients with PDAC vs patients with chronic pancreatitis. (A) Feature finding results showing the percentage occurrence of cytokines in models following resampling of training data for PDAC vs. chronic pancreatitis. (B) Training set ROC Curves for PDAC vs. chronic pancreatitis for a panel of IL-8, CA19-9, IL-6, IP-10 versus CA19-9 alone. (C) Test set ROC curves for PDAC vs. chronic pancreatitis for the panel of IL-8, CA19-9, IL-6 and IP-10 and CA19-9 alone. (D) The resampling performance of the panel of IL-8, CA19-9, IL-6, IP-10 versus CA19-9 for the classification of PDAC Vs. chronic pancreatitis. The accuracies, sensitivities and specificities of the panel compared to CA19-9 alone over 100 patient-balanced resamples is shown. The quantiles of the performance indicators are summarised as boxplots with individual points superimposed and the significance of Friedman test comparisons is indicated $(* * *<<0.001)$.

benign disease $=9)$ also showed diagnostic improvement $(\mathrm{AUC}=0.857, \mathrm{CI}=0.699-1 \quad$ vs. $\mathrm{AUC}=0.659, \mathrm{CI}=0.426$ 0.892 respectively) (Figure 4 C).

Resampling the combined dataset of patients with serum bilirubin levels $\geq 20 \mu \mathrm{mol} / \mathrm{L}$ ( $\mathrm{PDAC}=83$ and benign disease $=27$ ) generated 100 independent optimum values and showed significantly greater accuracy with the panel over CA19-9 $($ median $=73.9 \%, \mathrm{IQR}=68.5-80.8 \%$ vs. median $=$ $63.7 \%, \mathrm{IQR}=60.3-75.3 \%$, respectively; Friedman test $\mathrm{p}<$ 0.001) (Figure 4D). In particular, the $\mathrm{SN}$ for the cytokine panel was significantly higher than that of CA19-9 alone ( median $=74.5 \%, \mathrm{IQR}=63.6-85.9 \%$ vs. median $=61.8$, $\mathrm{IQR}=54.5-85.4 \%$, respectively; Friedman test $\mathrm{p}=0.002$ ).

\section{Ability to detect resectable PDAC and advanced PDAC cases}

The study included samples from both resectable and advanced PDAC cases (Additional file 1: Table S1). Binomial logistic modelling did not identify cytokines that distinguished between these two disease categories in either the training or test sets. However, post hoc tests showed that advanced and resectable PDAC were equally likely to be detected in our models as there was no significant difference in the proportion of advanced and resectable cases detected in pooled training and test set data (Pearson chi squared test for equality of proportion of detections of advanced and resectable PDAC at $\mathrm{df}=1$; PDAC vs. $\mathrm{HC}, \mathrm{p}=0.997$; PDAC vs. Benign Disease, $\mathrm{p}=0.417$; PDAC vs. $\mathrm{CP}, \mathrm{p}=$ 0.704 and PDAC with obstructive jaundice vs. Benign Disease with obstructive jaundice, $\mathrm{p}=0.892$ ).

\section{Discussion}

The accurate diagnosis of PDAC against a complex range of primary secondary and even tertiary health care scenarios remains a major clinical challenge. More specifically the clinical settings in which biomarker panels 

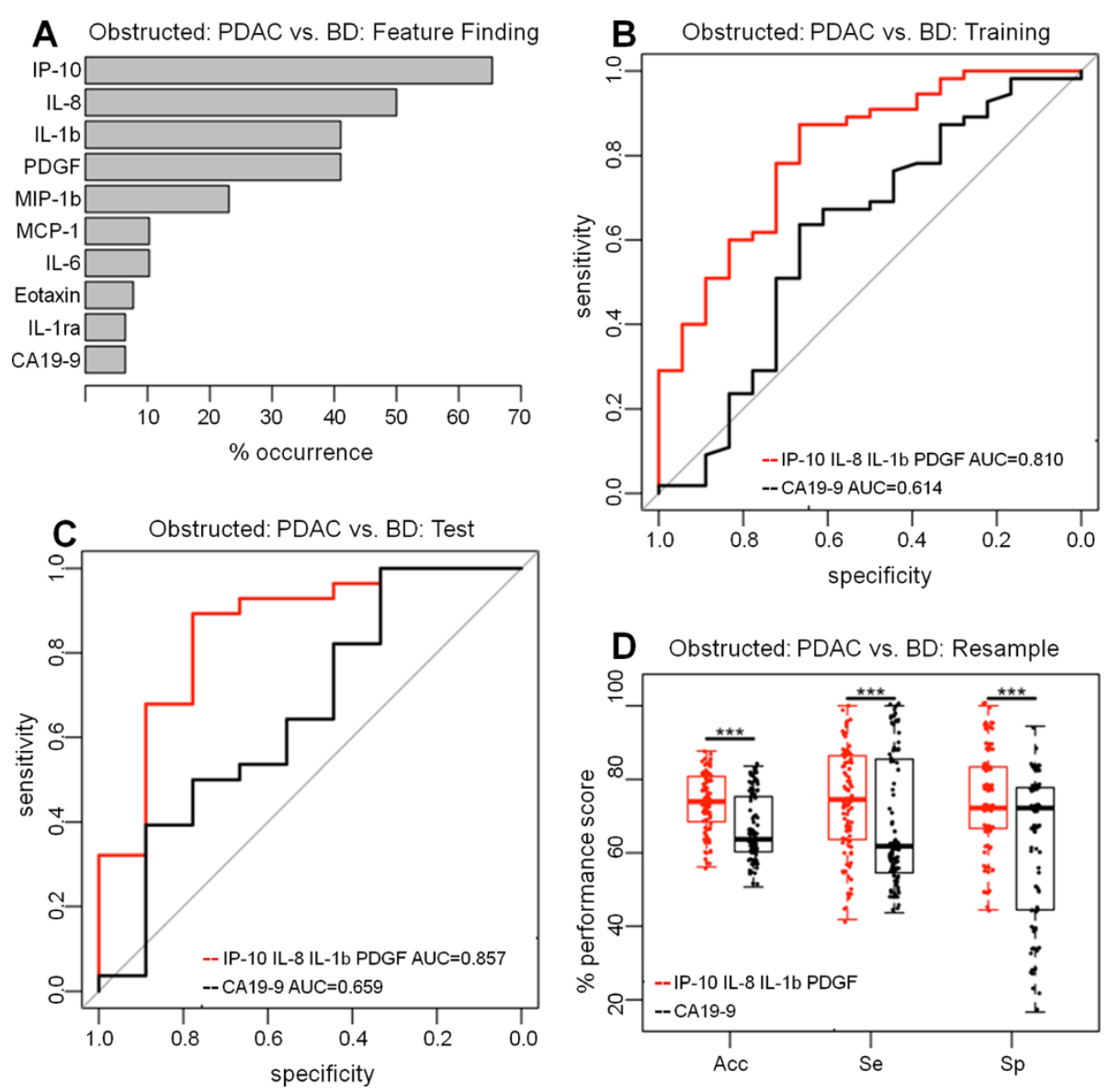

Figure 4 Classification of patients with PDAC in the presence of jaundice vs. patients with benign disease in the presence of jaundice. (A) Feature finding results showing the percentage occurrence of cytokines in models following resampling of training dataset for PDAC patients with high bilirubin vs. patients with benign disease and high bilirubin. (B) Training set ROC Curves for PDAC patients with high bilirubin vs. patients with benign disease and high bilirubin for a panel of IP-10, LL-8, IL-1b, PDGF and CA19-9 versus CA19-9 alone. (C) Test set ROC curves for PDAC patients with high bilirubin vs. patients with benign disease and high bilirubin for the panel of IP-10, IL-8, IL-1b, PDGF, CA19-9 compared to CA19-9 alone. (D) The resampling performance of the panel and CA19-9 for the classification of high bilirubin PDAC vs. high bilirubin benign disease. The accuracies, sensitivities and specificities of the panel IP-10, IL-8, IL-16, PDGF, CA19-9 compared to CA19-9 alone over 100 patient balanced resamples is shown. The quantiles of the performance indicators are summarised as boxplots with individual points superimposed and the significance of Friedman test comparisons is indicated $\left({ }^{* * *} p<<0.001\right)$.

may be used to facilitate accurate diagnosis are variable, depending on whether the disease is asymptomatic or symptomatic, and whether jaundice is present or absent. Thus, improving diagnosis involves discriminating patients with pancreatic cancer from patients with benign diseases of the pancreas or the biliary system. In this study, we used advance logistic modeling to determine how reliably distinct combinations of cytokines could facilitate differential pancreatic cancer diagnosis.

The performance of CA19-9 for the discrimination of PDAC patients from healthy subjects is variable, with some studies reporting just acceptable AUC values of $0.83-0.84[15,23]$, while one study an accuracy as high as $\mathrm{AUC}=0.90$ [24]. In this study we observed a very strong performance from CA19-9 compared to healthy controls, providing an AUC $>0.92$ in both training and test sets and very high median resampled estimates of optimum SN and SP were $85.9 \%$ and $96.3 \%$ respectively. The addition of two cytokines (IL-8 and IL-1b) to CA19-9 in a panel enhanced the performance of CA199, increasing the median resampled accuracy from $89 \%$ to $95 \%$ while the median resampled SN and SP was increased to $94 \%$ and $100 \%$, respectively. This supports the finding by Ebrahimi et al. [25] who found that IL-8 was increased in serum from PDAC patients compared to healthy controls, as was IL-1ra and IL-6, although these cytokines did not form part of the final panel.

The cytokines IL-8, IP-10, IL-6 and PDGF emerged as the strongest candidates in discriminating patients with PDAC from patients with benign pancreatic disease and combining these cytokines with CA19-9 afforded enhanced discrimination. The inconsistent outcomes with the training and test set data in this case illustrate the potential problems of overfitting and bias associated 
with single data splits, and supports the use of resampling for a more robust estimate of performance. It is notable that the SN and SP estimates with CA19-9 during resampling were bi-modal while the panel-derived estimates were uniformly distributed (Figure 2D). This suggested that the performance of CA19-9 alone was less uniform and therefore less reliable than the performance of the cytokine panel. Interestingly, a similar bi-modal distribution, resulting in an increase in the range of SN and SP estimates with CA19-9 compared to cytokine panels, was observed with resampled PDAC versus CP (Figure 3D), and with resampled High Bilirubin PDAC versus High Bilirubin Benign Disease patients (Figure 4D). This suggests that the panels in general provided a more reliable performance than CA19-9 alone.

Whilst CA19-9 levels were significantly raised in patients with biliary obstruction as might be expected [10,26,27] the levels of individual cytokines in cancer patients were not associated with jaundice. In discriminating jaundiced patients with PDAC from jaundiced patients with benign pancreatic disease, CA19-9 alone, performed poorly. IP-10, IL-8, IL-1b and PDGF, which were all significantly elevated in jaundiced patients with PDAC compared to jaundiced patients with benign disease, enabled significantly better discrimination of these two groups when used in combination. Even though obstructive jaundice occurs relatively late in pancreatic cancer, this can wane so more efficient and accurate differential diagnosis of these patients is of considerable importance in routine clinical practice. IL-8 levels are elevated in PDAC patients $[15,28]$ and this pro-inflammatory cytokine was a prominent feature as a discriminator for PDAC in all of the panels in this study. It is produced in monocytes and endothelial cells, and a variety of tumours [28-30] and high serum levels of IL- 8 in PDAC are linked to poor survival [31].

IP-10 featured in all disease comparison panels for diagnosis, consisitent with a previous small study in PDAC [32] and a study in colorectal cancer [33]. Increased expression of IP-10 and its receptor CXCR3 have also been associated with several advanced human cancers, including ovarian cancer, malignant melanoma, mutliple myeloma and basal cell carcinoma [34]. Whilst IP-10 generally performed well in the discrimination of PDAC from patients with benign disease, it was the best performing analyte for the discrimination of PDAC patients with jaundice from patients with benign disease in the presence of jaundice. This pro-inflammatory chemokine has also been shown to be secreted from several cell types in response to IFN- $\gamma$, and attracts activated lymphocytes, monocytes and NK cells to sites of inflammation [34], inhibits angiogenesis and promotes the survival and proliferation of tumour-specific T-cells [34-36].

IL-6 levels were observed to be significantly elevated in patients with PDAC compared to healthy controls, although it did not perform well enough to be part of the cytokine panel $[25,37,38]$. This cytokine was important in the discrimination of PDAC from chronic pancreatitis where it was the third most important analyte after IL-8 and CA19-9, during feature finding. It had limited value in distinguishing PDAC patients from patients with jaundice due to benign disease. IL-6 is a proimflamatory cytokine that it involoved in the recruitment of neutrophils and stimulating T-cell proliferation and migration [39]. High serum levels of IL-6 have been shown in many different cancer types, and positive associations with tumour stage, size and disease progression have been reported [39]. PDGF levels were not significantly different between patients with PDAC, patients with chronic pancreatitis and healthy subjects. Significantly decreased serum levels of PDGF were observed in patients with jaundice due to benign disease and may account for the discrimination of cancer patients from patients with biliary obstruction.

Other groups have examined the ability of cytokine panels to detect PDAC. Zeh et al. [40] used LabMAP serum technology with classification trees to identify panels to distinguish pancreatic cancer patients from chronic pancreatitis patients or control subjects. Interestingly their study identified IP-10 and IL-8 as having ability to discriminate PDAC from these two groups [40]. This is consistent with our study in which IL-8 was the best performing cytokine to distinguish PDAC cases from healthy controls and IP-10 and IL-8 were both featured in models distinguishing pancreatic cancer from benign disease. More recently, Dima et al. [31] explored the levels of circulating inflammatory cytokines in a small number of pancreatic cancer patients and controls. The study identified high levels of IL-6 in PDAC cases compared to chronic pancreatitis patients and elevated IL-10 and TNF $\alpha$ in PDAC cases compared to healthy subjects [31].

This study has a number of limitations. The sample sizes for investigating the effect of obstructive jaundice on performance were small, especially in the test datasets. In addition, the study suffered the loss of a number of analytes due to large coefficients of variance. Analytes such as IL-2, IL-15, and MIP-1a were present at very low concentrations and undetectable in more than half of the subjects studied. As expected the coefficient of variation was higher for analytes measured at lower concentrations on the Luminex platform compared to those measured at higher concentrations [41]. The diagnostic potential of these analytes should be tested under conditions sensitive to lower concentrations. Finally, we have confined this study to evaluating the potential of serum cytokine panels for pancreatic cancer diagnosis. The relationship between serum cytokine levels and prognosis is worthy of study, although lies outside the scope of this manuscript. 


\section{Conclusions}

In summary, we show that for the discrimination of patients with PDAC from patients with benign disease, combining IL-8, IP-10, IL-6 and PDGF with CA19-9 was better than using CA19-9 alone. Moreover, whilst CA199 was ineffective at discriminating between jaundiced PDAC patients versus jaundiced controls, a panel containing IP-10, IL-8, IL-1b and PDGF provided good discrimination. These findings support the potential role for specific cytokines in the differential diagnosis of pancreatic cancer and warrants additional study.

\section{Methods}

Blood samples using standard operating procedures were obtained from pre-surgical resection (resectable) or by-pass (advanced) patients with histologically confirmed PDAC, histologically confirmed chronic pancreatitis $(\mathrm{CP})$, benign biliary obstruction (BBO) or from healthy controls (HC). Resectable PDAC patients had normal tissue plane between tumour and vessels and no evidence of metastatic disease or tumour abutment less than $180^{\circ}$ of the SMA or coeliac axis, venous involvement up to $2 \mathrm{~cm}$ occlusion of the SMV, PV or SMV-PV confluence with no evidence of metastatic disease [42-44]. All patients gave written informed consent using approved ethics protocols, at the Royal Liverpool University Hospital.

\section{Serum collection}

Blood was collected in Sarstedt Monovette Serum Z tubes (Sarstedt Ltd, Leicester, UK) and allowed to coagulate for 30 minutes before centrifugation at $800 \times \mathrm{g}$ for $10 \mathrm{~min}$. The serum fraction was aliquotted into cryotubes and stored at $-80^{\circ} \mathrm{C}$. CA19-9 levels were measured using ELISA (Human Pancreatic \& GI Cancer ELISA Kit, Alpha Diagnostics International, San Antonio, Tx, USA). Pre-operative total serum bilirubin $(\mu \mathrm{mol} / \mathrm{L})$ (Roche Modular SWA) was measured in the hospital Clinical Biochemistry Department.

\section{Measurement of cytokines}

The serum levels of 27 cytokines, chemokines and growth factors from patients and healthy subjects were measured blindly in duplicate using a commercially available Bio-Plex Pro 27 Plex Human Cytokine, Chemokine and Growth Factor Assay (Bio-Rad Laboratories Ltd, Hercules, CA, USA), on the Bio-Plex 200 System, with initial data analysis to measure concentration performed using Bio-Plex Manager 5.0 Software. Briefly, serially diluted standards $(50 \mu \mathrm{L})$ and test serum, diluted 1 in 4 in sample diluent, $(50 \mu \mathrm{l})$ was added to a microfilter plate containing antibody-coupled beads for each of the 27 analytes. The microfilter plate was incubated at room temperature on a plate shaker at $900 \mathrm{rpm}$ for $1 \mathrm{mi}-$ nute followed by $300 \mathrm{rpm}$ for 30 minutes. Following washing by vacuum filtration the secondary antibodies $(25 \mu \mathrm{L})$ were added and the microfilter plate incubated as before. The microfilter plate was washed again and Streptavidin-PE $(50 \mu \mathrm{L})$ was added and the plate incubated at room temperature on a plate shaker at $900 \mathrm{rpm}$ for 1 minute followed by $300 \mathrm{rpm}$ for 15 minutes. Assay buffer $(125 \mu \mathrm{L})$ was added to each well of the microfilter plate before being read on the Bio-Plex 200 machine. Fluorescent intensities obtained for the test samples were read from the standard curve to give $\mathrm{pg} / \mathrm{mL}$ values for each of the 27 cytokines, chemokines and growth factors. Ten assay plates were used to generate training data from 158 individuals, and test data from 83 individuals. To assess inter-plate variation, 6 individual samples were measured across triplicate plates; and at least one aliquot of the same PDAC patient was assayed on every plate for internal control purposes.

\section{Patient groups}

The training set consisted of samples from 158 subjects, 84 patients with PDAC, 45 patients with benign pancreatic disease (32 with $\mathrm{CP}$ and 13 with $\mathrm{BBO}$ due to gall stones) and 29 HCs. The serum bilirubin level of patients was recorded in all cases as either low $(<20 \mu \mathrm{mol} /$ $\mathrm{L}$; upper level of normal for our Centre) or high $(>20 \mu \mathrm{mol} / \mathrm{L})$. In the training set there were $73(46.2 \%)$ patients with high bilirubin, 55 with PDAC and 18 with $\mathrm{BBO}$ including 5 with $\mathrm{CP}$. The independent test set consisted of samples from 83 subjects, 43 patients with PDAC, 17 with $\mathrm{CP}, 7$ with $\mathrm{BBO}$, and $16 \mathrm{HCs}$. In the test set there were $37(44.6 \%)$ patients with high bilirubin, 28 with PDAC and 9 patients with BBO (including 2 with $\mathrm{CP})$. The clinical characteristics of the training and test study populations are provided in Table 3, with further specific characteristics of cancer patients, separated into resectable and advanced categories, provided in Additional file 2: Table S2. The median age of healthy control subjects in the training set was 44 years compared to a median age of 66 years for the PDAC patients and the median age of healthy control subjects in the test set was higher at 56.5 years.

\section{Data filtering, normalisation}

Cytokines with internal control measurements with a coefficient of variance $>50 \%$ were removed from the dataset. Cytokine concentrations $(\mathrm{pg} / \mathrm{mL})$ less than the lower limit of detection were set to 0.001 . The remaining cytokines, along with CA19-9, were used in predictor model building. Normalisation between plates was undertaken by dividing raw cytokine data by the plate-specific internal control value for each cytokine. Normalised data were $\log _{2}$ transformed for statistical analysis. The Shapiro-Wilk test with cut-off of $\mathrm{p}<0.05$ was applied to test the null hypothesis that data were normally distributed. Two-sample Wilcoxon 
Table 3 Clinical characteristics of respective training and test study populations

\begin{tabular}{|c|c|c|c|c|}
\hline $\begin{array}{l}\text { Training set } \\
\mathrm{n}=158\end{array}$ & $\begin{array}{l}\text { Age (years) } \\
\text { Median }(95 \% \mathrm{Cl}) \\
\end{array}$ & $\begin{array}{l}\text { Gender } \\
\text { Male: Female }\end{array}$ & $\begin{array}{l}\text { CA19-9 }(\mathrm{KU} / \mathrm{L}) \\
\text { Median }(95 \% \mathrm{Cl})\end{array}$ & $\begin{array}{l}\text { Bilirubin }(\mu \mathrm{mol} / \mathrm{L}) \\
\text { Median }(95 \% \mathrm{Cl}) \\
\end{array}$ \\
\hline PDAC $(n=84)$ & $66(64.0-69.0)$ & $47: 37$ & $137.5(62.0-161.4)$ & $31(24.5-54.3)$ \\
\hline$C P(n=32)$ & $53(48.0-58.6)$ & $20: 12$ & $24.5(11.0-41.0)$ & $7(6.0-10.0)$ \\
\hline BBO $(n=13)$ & $72(60.7-77.4)$ & $11: 2$ & $89(13.5-228.3)$ & $69(11.0-108.6)$ \\
\hline$H C(n=29)$ & 44 (29.9-54.0) & 14:15 & $4(2.0-6.0)$ & NA \\
\hline $\begin{array}{l}\text { Test Set } \\
\mathrm{n}=83\end{array}$ & $\begin{array}{l}\text { Age (years) } \\
\text { Median }(95 \% \mathrm{Cl})\end{array}$ & $\begin{array}{l}\text { Gender } \\
\text { Male: Female }\end{array}$ & $\begin{array}{l}\text { CA19-9 (KU/L) } \\
\text { Median }(95 \% \mathrm{Cl})\end{array}$ & $\begin{array}{l}\text { Bilirubin }(\mu \mathrm{mol} / \mathrm{L}) \\
\text { Median }(95 \% \mathrm{Cl})\end{array}$ \\
\hline PDAC $(n=43)$ & $68(64.2-71.8)$ & $24: 19$ & $116(54.9-164.4)$ & $51(19.6-114.0)$ \\
\hline$C P(n=17)$ & $53(45.1-62.0)$ & $10: 7$ & $12(8.0-24.9)$ & $6(5.0-10.0)$ \\
\hline BBO $(n=7)$ & $64(38.4-78.5)$ & $3: 4$ & $37(4.5-181.3)$ & $100(44.9-374.9)$ \\
\hline$H C(n=16)$ & $56.5(36.7-62.1)$ & 9:7 & $3(2.6-8.4)$ & NA \\
\hline
\end{tabular}

$\mathrm{PDAC}=$ Cancer, $\mathrm{CP}=$ Chronic Pancreatitis, $\mathrm{BBO}=$ Benign Biliary Obstruction, $\mathrm{HC}=$ Healthy Controls.

signed rank (Mann-Whitney) tests with a cut-off of $\mathrm{p}<$ 0.05 were applied to test the null-hypothesis that the medians of groups with non-parametric (non-normal) distribution were the same. Following normalisation and $\log _{2}$ transformation, the class labels (e.g. PDAC, CP, BBO or $\mathrm{HC}$ ) were added to the data set.

\section{Building statistical predictor models}

Statistical modelling was performed in 64-bit R (2.15.1; $\mathrm{R}$ Development Core Team 2012). The training dataset was used to construct binomial logistic models, using the $\mathrm{R}$ function glm, to discriminate the following patient groups: PDAC versus $\mathrm{HC}$; PDAC versus Benign Disease (combined $\mathrm{CP}$ and $\mathrm{BBO}$ patients) PDAC versus $\mathrm{CP}$ and $\mathrm{PDAC}$ in the presence of jaundice versus Benign Disease in the presence of jaundice.

Cytokines for inclusion in models were selected using repeated $(n=100)$ balanced splitting of the training set, tabulating the optimal cytokine panel for each model and then ranking cytokines. Feature finding at each data split was performed by complete enumeration of all possible logistic regression models by Morgan-Tatar search, selecting the highest ranked model as scored by Bayesian information criterion using the bestglm package in R. Cytokines were selected if they achieved a regression $\mathrm{p}<0.05$ in the top ranked model. Logistic predictor models fitted to data in this way consisted of a probability function (i.e. a predictor of class membership) of the form $\exp (z) / 1+\exp (z)$ where $z$ is a specific linear combination of coefficients, such that $z=\beta_{0}+\beta_{1} x_{1}+\beta_{2} x_{2} \ldots$, where $\beta_{i}$ are cytokine specific coefficients found by regression ( $\beta_{o}$ being the intercept) and $x_{i}$ are sample cytokine specific values. Classification performance was assessed by Receiver Operating Characteristic (ROC) analysis using the pROC [45] and ROCR [46] packages from $\mathrm{R}$ to find the optimum sensitivity ( $\mathrm{SN}$ ), specificity (SP) and Area Under Curve (AUC). Comparisons of AUCs of cytokine panel models versus CA19-9 alone were performed using a one-tailed De Long's test. The classification performance of models developed with the training data was assessed by internal validation and independently using the test set data. Confidence intervals (95\%) for the SN and SP of individual diagnostic tests were calculated as exact binomial proportion intervals using the $\mathrm{R}$ package epiR. The McNemar test was used to compare SN and SP of paired diagnostic tests using the R package DTComPair [47].

\section{Resampled estimates of model performance}

The robustness of the classification models developed with training data was evaluated by repeatedly resampling data from the combined training and test cohorts. The combined dataset was split randomly 100 times at a ratio of 2 to 1 into resampled training and test sets, taking care to maintain the proportionate class composition of the resampled training and test sets (i.e. the ratio of cancer: control). At each split, the resampled training dataset was used to build a classification model using the cytokine panel identified during binomial logistic modelling of the original training sets. The ability of each model to classify the resampled test data was recorded. ROC analysis was performed and the SN and SP values, at the optimum accuracy, of each resampled test set were recorded. Thus, each resampled model generated 100 independent estimates of $\mathrm{SN}, \mathrm{SP}$ at optimised accuracy, which were used to calculate median SN/SP and median accuracy values. Since the distribution of prediction error of resampled models was non-normal and prediction errors with the same test set but different models are not independent, the null hypothesis of equality of prediction error distribution of different models was tested using the Friedman test.

\section{Additional files}

Additional file 1: Table S1. Circulating cytokine levels in the combined training and test datasets.

Additional file 2: Table S2. Patient characteristics of Resectable and Advanced PDAC patients. 


\section{Competing interests}

The authors declare that they have no competing interests.

\section{Authors' contributions}

VES and CJ carried out cytokine luminex analysis of patient serum samples. VES, EC and BL performed statistical and bioinformatics analysis of cytokine data. TC advised on statistical analysis. VES and EC conceived of the experiments. All authors participated in study design, coordination and helped to draft the manuscript. All authors read and approved the final manuscript.

\section{Acknowledgments}

This work was supported by The National Institute for Health Research Pancreas Biomedical Research Unit, Cancer Research UK grant A12790 and Pancreatic Cancer UK.

Received: 18 December 2013 Accepted: 23 April 2014

Published: 20 May 2014

\section{References}

1. Costello E, Greenhalf W, Neoptolemos JP: New biomarkers and targets in pancreatic cancer and their application to treatment. Nat Rev Gastroenterol Hepatol 2012, 9:435-444.

2. Costello E, Neoptolemos JP: Pancreatic cancer in 2010: new insights for early intervention and detection. Nat Rev Gastroenterol Hepatol 2011, 8:71-73.

3. Martin KJ, Fournier MV, Reddy GP, Pardee AB: A need for basic research on fluid-based early detection biomarkers. Cancer Res 2010, 70:5203-5206.

4. Locker GY, Hamilton S, Harris J, Jessup JM, Kemeny N, Macdonald JS, Somerfield MR, Hayes DF, Bast RC Jr: Asco: ASCO 2006 update of recommendations for the use of tumor markers in gastrointestinal cancer. J Clin Oncol 2006, 24:5313-5327.

5. Wong D, Ko AH, Hwang J, Venook AP, Bergsland EK, Tempero MA: Serum CA19-9 decline compared to radiographic response as a surrogate for clinical outcomes in patients with metastatic pancreatic cancer receiving chemotherapy. Pancreas 2008, 37:269-274.

6. Winter JM, Yeo CJ, Brody JR: Diagnostic, prognostic, and predictive biomarkers in pancreatic cancer. J Surg Oncol 2013, 107:15-22.

7. Sener SF, Fremgen A, Menck HR, Winchester DP: Pancreatic cancer: a report of treatment and survival trends for 100,313 patients diagnosed from 1985-1995, using the National cancer database. J Am Coll Surg 1999, 189:1-7

8. Singh S, Tang SJ, Sreenarasimhaiah J, Lara LF, Siddiqui A: The clinical utility and limitations of serum carbohydrate antigen (CA19-9) as a diagnostic tool for pancreatic cancer and cholangiocarcinoma. Dig Dis Sci 2011 56:2491-2496

9. Hamada S, Shimosegawa T: Biomarkers of pancreatic cancer. Pancreatology 2011, 11(Suppl 2):14-19.

10. Smith RA, Bosonnet $L$, Ghaneh P, Raraty M, Sutton R, Campbell F, Neoptolemos JP: Preoperative CA19-9 levels and lymph node ratio are independent predictors of survival in patients with resected pancreatic ductal adenocarcinoma. Dig Surg 2008, 25:226-232.

11. von Ritter C, Eder MI, Stieber P, Lamerz R, Jungst D, Strigl M, Meyer G, Reuter C, Paumgartner G: Biliary mucin secreted by cultured human gallbladder epithelial cells carries the epitope of CA 19-9. Anticancer Res 1997 17:2931-2934.

12. Wingren C, Sandstrom A, Segersvard R, Carlsson A, Andersson R, Lohr M, Borrebaeck CA: Identification of serum biomarker signatures associated with pancreatic cancer. Cancer Res 2012, 72:2481-2490.

13. Park HD, Kang ES, Kim JW, Lee KT, Lee KH, Park YS, Park JO, Lee J, Heo JS, Choi SH, Choi DW, Kim S, Lee JK, Lee SY: Serum CA19-9, cathepsin D, and matrix metalloproteinase-7 as a diagnostic panel for pancreatic ductal adenocarcinoma. Proteomics 2012, 12:3590-3597.

14. Faca VM, Song KS, Wang H, Zhang Q, Krasnoselsky AL, Newcomb LF, Plentz RR, Gurumurthy S, Redston MS, Pitteri SJ, Pereira-Faca SR, Ireton RC, Katayama H, Glukhova V, Phanstiel D, Brenner DE, Anderson MA, Misek D, Scholler N, Urban ND, Barnett MJ, Edelstein C, Goodman GE, Thornquist MD, Mclntosh MW, DePinho RA, Bardeesy N, Hanash SM: A mouse to human search for plasma proteome changes associated with pancreatic tumor development. PLoS Med 2008, 5:e123.
15. Brand RE, Nolen BM, Zeh HJ, Allen PJ, Eloubeidi MA, Goldberg M, Elton E, Arnoletti JP, Christein JD, Vickers SM, Langmead CJ, Landsittel DP, Whitcomb DC, Grizzle WE, Lokshin AE: Serum biomarker panels for the detection of pancreatic cancer. Clin Cancer Res 2011, 17:805-816.

16. Pan S, Chen R, Crispin DA, May D, Stevens T, Mclntosh MW, Bronner MP, Ziogas A, Anton-Culver $\mathrm{H}$, Brentnall TA: Protein alterations associated with pancreatic cancer and chronic pancreatitis found in human plasma using global quantitative proteomics profiling. J Proteome Res 2011, 10:2359-2376.

17. Bauer AS, Keller A, Costello E, Greenhalf W, Bier M, Borries A, Beier M, Neoptolemos J, Buchler M, Werner J, Giese N, Hoheisel JD: Diagnosis of pancreatic ductal adenocarcinoma and chronic pancreatitis by measurement of microRNA abundance in blood and tissue. PLoS One 2012, 7:e34151.

18. Tonack S, Aspinall-O'Dea M, Neoptolemos JP, Costello E: Pancreatic cancer: proteomic approaches to a challenging disease. Pancreatology 2009, 9:567-576.

19. Blanchet $X$, Langer M, Weber $C$, Koenen RR, von Hundelshausen P: Touch of chemokines. Front Immunol 2012, 3:175.

20. Evans $A$, Costello $E$ : The role of inflammatory cells in fostering pancreatic cancer cell growth and invasion. Front Physiol 2012, 3:270

21. Schetter AJ, Heegaard NH, Harris CC: Inflammation and cancer: interweaving microRNA, free radical, cytokine and $\mathrm{p} 53$ pathways. Carcinogenesis 2010, 31:37-49.

22. Dudoit S, Fridlyand J, Speed TP: Comparison of discrimination methods for the classification of tumors using gene expression data. J Am Stat Assoc 2002, 97:77-87.

23. Joergensen MT, Brunner N, De Muckadell OB: Comparison of circulating MMP-9, TIMP-1 and CA19-9 in the detection of pancreatic cancer. Anticancer Res 2010, 30:587-592.

24. Xue A, Scarlett CJ, Chung L, Butturini G, Scarpa A, Gandy R, Wilson SR, Baxter RC, Smith RC: Discovery of serum biomarkers for pancreatic adenocarcinoma using proteomic analysis. Br J Cancer 2010, 103:391-400.

25. Ebrahimi B, Tucker SL, Li D, Abbruzzese JL, Kurzrock R: Cytokines in pancreatic carcinoma: correlation with phenotypic characteristics and prognosis. Cancer 2004, 101:2727-2736.

26. Yan L, Tonack S, Smith R, Dodd S, Jenkins RE, Kitteringham N, Greenhalf W Ghaneh P, Neoptolemos JP, Costello E: Confounding effect of obstructive jaundice in the interpretation of proteomic plasma profiling data for pancreatic cancer. J Proteome Res 2009, 8:142-148.

27. Tonack S, Jenkinson C, Cox T, Elliott V, Jenkins RE, Kitteringham NR, Greenhalf W, Shaw V, Michalski CW, Friess H, Neoptolemos JP, Costello E: ITRAQ reveals candidate pancreatic cancer serum biomarkers: influence of obstructive jaundice on their performance. Br J Cancer 2013, 108:1846-1853.

28. Chen Y, Shi M, Yu GZ, Qin XR, Jin G, Chen P, Zhu MH: Interleukin-8, a promising predictor for prognosis of pancreatic cancer. World $J$ Gastroenterol 2012, 18:1123-1129.

29. Xie K: Interleukin-8 and human cancer biology. Cytokine Growth Factor Rev 2001, 12:375-391.

30. Hussain F, Wang J, Ahmed R, Guest SK, Lam EW, Stamp G, El-Bahrawy M: The expression of IL- 8 and IL-8 receptors in pancreatic adenocarcinomas and pancreatic neuroendocrine tumours. Cytokine 2010, 49:134-140.

31. Dima SO, Tanase C, Albulescu R, Herlea V, Chivu-Economescu M, Purnichescu-Purtan R, Dumitrascu T, Duda DG, Popescu I: An exploratory study of inflammatory cytokines as prognostic biomarkers in patients with ductal pancreatic adenocarcinoma. Pancreas 2012, 41:1001-1007.

32. Bellone G, Novarino A, Chiappino I, Gramigni C, Carbone A, Addeo A, Tonel E, Bertetto O, Emanuelli G: Circulating vascular endothelial growth factor and interferon-gamma-inducible protein-10 levels in pancreatic cancer during chemotherapy. Anticancer Res 2005, 25:3287-3291.

33. Toiyama Y, Fujikawa H, Kawamura M, Matsushita K, Saigusa S, Tanaka K, Inoue Y, Uchida K, Mohri Y, Kusunoki M: Evaluation of CXCL10 as a novel serum marker for predicting liver metastasis and prognosis in colorectal cancer. Int J Oncol 2012, 40:560-566.

34. Liu M, Guo S, Stiles JK: The emerging role of CXCL10 in cancer (Review). Oncol Lett 2011, 2:583-589.

35. Mei K, Wang L, Tian L, Yu J, Zhang Z, Wei Y: Antitumor efficacy of combination of interferon-gamma-inducible protein 10 gene with gemcitabine, a study in murine model. J Exp Clin Cancer Res 2008, 27:63.

36. Yang $X$, Chu Y, Wang Y, Zhang R, Xiong S: Targeted in vivo expression of IFN-gamma-inducible protein 10 induces specific antitumor activity. J Leukoc Biol 2006, 80:1434-1444. 
37. Barber MD, Fearon KC, Ross JA: Relationship of serum levels of interleukin6, soluble interleukin-6 receptor and tumour necrosis factor receptors to the acute-phase protein response in advanced pancreatic cancer. Clin Sci (Lond) 1999, 96:83-87.

38. Bartsch R, Woehrer S, Raderer M, Hejna M: Serum interleukin-6 levels in patients with gastric MALT lymphoma compared to gastric and pancreatic cancer. Anticancer Res 2006, 26:3187-3190.

39. Lippitz BE: Cytokine patterns in patients with cancer: a systematic review. Lancet Oncol 2013, 14:e218-228.

40. Zeh HJ, Winikoff S, Landsittel DP, Gorelik E, Marrangoni AM, Velikokhatnaya L, Winans MT, Lee K, Moser A, Bartlett D, Lotze MT, Siegfried JM, Whitcomb D, Papacristou G, Slivka A, Bigbee WL, Lokshin AE: Multianalyte profiling of serum cytokines for detection of pancreatic cancer. Cancer Biomark 2005, 1:259-269.

41. Chowdhury F, Williams A, Johnson P: Validation and comparison of two multiplex technologies, Luminex and Mesoscale Discovery, for human cytokine profiling. J Immunol Methods 2009, 340:55-64.

42. Varadhachary GR, Tamm EP, Abbruzzese $J \mathrm{~L}$, Xiong HQ, Crane CH, Wang $\mathrm{H}$, Lee JE, Pisters PW, Evans DB, Wolff RA: Borderline resectable pancreatic cancer: definitions, management, and role of preoperative therapy. Ann Surg Oncol 2006, 13:1035-1046.

43. Choti MA, Dixon E, Tyler D: Pretreatment assessment of resectable and borderline resectable pancreatic cancer: expert consensus statement by Callery et al. Ann Surg Oncol 2009, 16:1734-1735.

44. Callery MP, Chang K, Fishman EK, Talamonti MS, William Traverso L, Linehan DC: Pretreatment assessment of resectable and borderline resectable pancreatic cancer: expert consensus statement. Ann Surg Oncol 2009, 16:1727-1733.

45. Robin X, Turck N, Hainard A, Tiberti N, Lisacek F, Sanchez JC, Muller M: pROC: an open-source package for $\mathrm{R}$ and $\mathrm{S}+$ to analyze and compare ROC curves. BMC Bioinforma 2011, 12:77.

46. Sing T, Sander O, Beerenwinkel N, Lengauer T: ROCR: visualizing classifier performance in R. Bioinformatics 2005, 21:3940-3941.

47. Hothorn T, Leisch F, Zeileis A, Hornik K: The design and analysis of benchmark experiments. J Comput Graph Stat 2005, 14:675-699.

doi:10.1186/1476-4598-13-114

Cite this article as: Shaw et al.: Serum cytokine biomarker panels for discriminating pancreatic cancer from benign pancreatic disease.

Molecular Cancer 2014 13:114.

\section{Submit your next manuscript to BioMed Central and take full advantage of:}

- Convenient online submission

- Thorough peer review

- No space constraints or color figure charges

- Immediate publication on acceptance

- Inclusion in PubMed, CAS, Scopus and Google Scholar

- Research which is freely available for redistribution

Submit your manuscript at www.biomedcentral.com/submit
C Biomed Central 